\title{
Does a text-messaging program to promote early childhood development reach the highest risk families?
}

\author{
Maureen Cunningham ${ }^{1,2}$, Sheana Bull ${ }^{3}$, Monica C. McNulty ${ }^{4}$, Kathryn Colborn ${ }^{3}$, Catia Chavez ${ }^{4}$, \\ Stephen Berman ${ }^{1,2}$, Jean McSpadden ${ }^{5}$, Jared Wigdor ${ }^{5}$, Mandy A. Allison ${ }^{1,4}$ \\ ${ }^{1}$ Department of Pediatrics, University of Colorado Denver School of Medicine, Aurora, CO, USA; ${ }^{2}$ Center for Global Health, Colorado School of \\ Public Health, Aurora, CO, USA; ${ }^{3}$ Colorado School of Public Health, Anschutz Medical Campus, Aurora, CO, USA; ${ }^{4}$ Adult and Child Consortium \\ for Health Outcomes Research and Delivery Science, Aurora, CO, USA; ${ }^{5}$ Bright by Three, Denver, CO, USA \\ Contributions: (I) Conception and design: M Cunningham, S Bull, MC McNulty, K Colborn, S Berman, MA Allison; (II) Administrative support: \\ C Chavez, J Wigdor; (III) Provision of study materials or patients: J Wigdor, J McSpadden; (IV) Collection and assembly of data: MC McNulty, \\ K Colborn, J Wigdor; (V) Data analysis and interpretation: K Colborn, MC McNulty, M Cunningham, MA Allison; (VI) Manuscript writing: \\ All authors; (VII) Final approval of manuscript: All authors. \\ Correspondence to: Maureen Cunningham, MD, MPH. 13199 Montview Blvd. A090, Ste 310, Aurora, CO 80045, USA. \\ Email: Maureen.Cunningham@ucdenver.edu.
}

Background: Bright by Three (BB3), a non-profit organization that promotes caregiver behaviors to support language development in young children was augmented with a text-messaging program, Bright by Text (BBT), in 2015. While some evidence suggests that text-messaging can promote early development, it is unknown if these interventions are reaching children at increased sociodemographic risk for developmental delay. The purpose of this study is to compare socio-demographic characteristics of caregivers who did and did not enroll in BBT.

Methods: Retrospective analysis of caregivers who received BB3 written materials and were eligible to sign up for BBT in 2016. Outcomes: (I) provision of a mobile phone number; (II) enrollment in BBT (receipt of $3+$ messages). Predictors: education, marital status, race/ethnicity, insurance, language, and urban $v s$. rural residence. A multivariable generalized linear model was used to determine characteristics of caregivers more likely to sign up for BBT.

Results: A total of 18,145 caregivers received BB3 written materials; 10,843 (60\%) provided a mobile phone number and 2,314 (21\%) enrolled in BBT. The relative risk (RR) of enrollment was higher for caregivers who were non-minority (RR 1.15, 95\% CI, 1.04-1.28), had higher education (1.60, 1.35-1.89), had private insurance $(1.15,1.15-1.28)$ and lived in urban areas $(1.21,1.06-1.37)$. Non-English speaking caregivers were less likely to enroll (0.73, 0.59-0.90).

Conclusions: Caregivers with lower incomes and education, minorities and non-English speakers were less likely to enroll in BBT. Future research could identify ways to increase engagement among these populations and determine if BBT is effective in changing parent behavior and improving children's development.

Keywords: Mobile health (mHealth); Short Messaging Service messaging (SMS messaging); text-messaging; early child development

Received: 10 October 2018; Accepted: 14 November 2018; Published: 29 November 2018.

doi: $10.21037 /$ mhealth.2018.11.03

View this article at: http://dx.doi.org/10.21037/mhealth.2018.11.03 


\section{Introduction}

In 1995 Hart and Risley found that low-income children heard an average of 30 million fewer words during the first 3 years of life compared to their higher income peers. Likewise, low-income children were less likely to hear words of praise and encouragement from their caregivers. These children had lower vocabulary and IQ scores at age 3 years and went on to have comparatively lower language skills at age 9-10 years (1). Indeed the "30-million-word gap" discovered by Hart and Risley has received much attention as child health and development experts grapple with how to narrow this divide. Other studies have found that low-income children go on to have poor school readiness (2), reduced academic success (3), lower adult earning potential and worse long-term health outcomes (4). The accumulation of stressors precipitated by poverty, neglect, and abuse is termed toxic stress, and this prolonged stress response has been found to have a significant impact on early brain development and architecture (5-9).

Research has shown that supportive and responsive relationships with caring adults early in life can prevent or reverse the effects of toxic stress and narrow the gaps in academic achievement, adult earning potential, and health outcomes faced by low-income children (10). Intensive center-based interventions as well as less intensive pediatric practice based interventions have been found to have positive impacts on long-term health related outcomes as well as short-term impacts on home environment and early language development (11-15). The reach of these programs, however is limited by cost and location making mobile health (mHealth) interventions an inexpensive and attractive alternative.

According to the PEW Research Center, in January $2017,92 \%$ of adults with income less than $\$ 30,000$ /year reported owning a cell phone and $64 \%$ reported owning a smartphone (16). There has been a proliferation of interventions to promote health that utilize text messaging or Short Messaging Service (SMS). This is encouraging in the face of a shrinking digital divide and increasing access to Internet-enabled or "smart" mobile phones, and suggests there are increasing opportunities to reach lower income and persons who face disparities in health outcomes with mobile and technology-based solutions (17). mHealth is the delivery of healthcare and public health interventions via mobile devices such as smart phones and tablets. With the potential to reach many different segments of the population at minimal cost, these interventions have a wide reach and can be easily scaled.
While there is a growing body of evidence that well designed mHealth interventions can impact health behavior and health outcomes, we know little about low-income populations' motivations to adopt mHealth solutions and whether the benefits of mHealth extend to low-income communities. For example, recent studies have found that text-messaging programs can increase home support for language development thus improving early literacy $(18,19)$. Less is understood, however, about whether these types of interventions are actually being adopted and used by lowincome, minority and non-English speaking caregivers. If these interventions are not reaching this high priority audience, it is difficult to study effectiveness in the target population. The purpose of the present study was to determine the reach of a text-messaging program designed to promote early child development by understanding the sociodemographic characteristics of caregivers who enrolled in the program. We hypothesized that caregivers with lower income and education, minorities, and non-English speakers would be less likely to enroll.

\section{Methods}

This is a retrospective analysis of caregivers who were involved with Bright by Three (BB3) in 2016. Approval for this secondary analysis of BB3 program data was obtained from the Colorado Multiple Institution Review Board (COMIRB).

\section{Description of the BB3 and BBT programs}

BB3 (formerly Colorado Bright Beginnings) was founded in 1995 to promote the well-being, of all of Colorado's children. The organization reaches more than 20,000 families each year. BB3 is focused on the promotion of responsive parenting and encourages caregivers to talk, read, play with and praise their young children. Materials are provided to caregivers of children ages $0-3$ years and include a picture book (20), parent handbook, Language Power (21) and Learning Games (22) These materials are designed to advise parents on how they can engage in activities to support their child's cognitive, language and social emotional development. BB3 makes special efforts to outreach to low income families via partnerships with medical clinics, hospitals, community organizations and home visitation programs.

Bright by Text (BBT) was initiated in 2015 to augment BB3's existing printed materials. BBT delivers free advice 


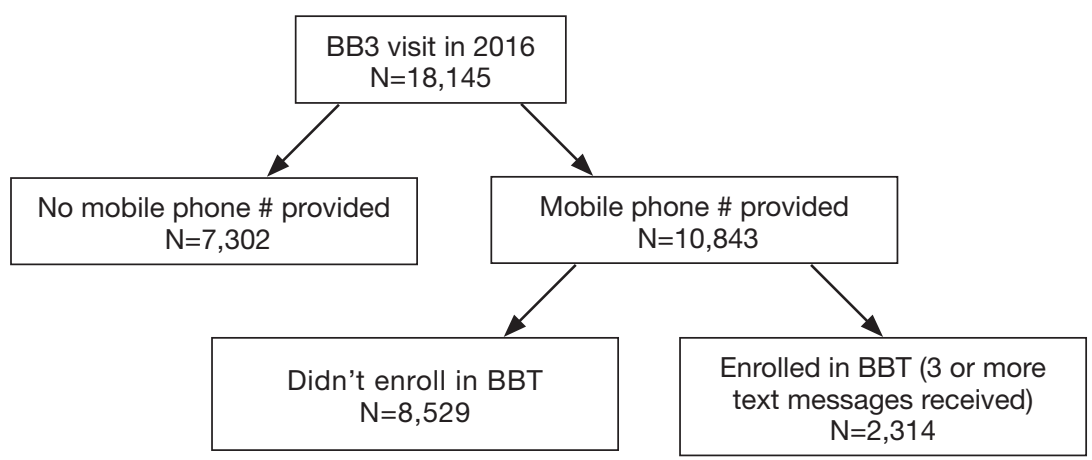

Figure 1 Caregivers receiving BB3 toolkit, providing mobile phone number, and enrolling in BBT in 2016. BB3, Bright by Three; BBT, Bright by Text.

about developmentally appropriate activities and games as well as information about local resources to caregivers via short and easy to read SMS messaging. Messages are written at a $6^{\text {th }}$ grade reading level and contain a link to a landing page where users can navigate for more information. Caregivers are invited to enroll in BBT via a postcard that is included in a toolkit with their BB3 written materials. BBT is available in English and Spanish.

\section{Data}

When caregivers received a BB3 toolkit they were asked to provide sociodemographic information including race/ ethnicity, education, insurance, language spoken at home, marital status and zip code. These data were used to estimate associations between BB3 and BBT participation and sociodemographic characteristics. Caregivers who identified as a race other than white or reported Hispanic or Latino ethnicity were considered minorities. BB3 does not collect data on household income but does document type of health insurance. Caregivers who reported that they had public health insurance (Medicaid or $\mathrm{CHP}+$ ) or were uninsured were considered lower income. Caregivers who reported that they had private insurance were considered higher income. BB3 also collected information on caregiver education. Caregivers were separated into one of 3 possible categories: those who did not graduate from high school, high school graduates and college graduates or higher. Marital status was also collected. Caregivers who reported that they were single, divorced or widowed were considered unmarried and those who reported that they were married or living with their partner were considered married in analyses. Caregivers who reported speaking only English or English and another language were considered English speakers. Caregivers who reported speaking only Spanish were non-English speakers. Population density was determined by the zip code caregivers provided at intake. When they received their $\mathrm{BB} 3$ packet, caregivers were also asked to provide a mobile phone number. If a mobile phone number was provided, we assumed that users had access to a mobile device and were able to receive messages.

\section{Outcome variables}

Outcome variables were provision of a mobile phone number upon receipt of a BB3 packet and enrollment in BBT. Enrollment was defined as receipt of 3 or more text messages which signals initiation of enrollment by the caregiver, response to a prompt to input their child's information and preferred language, a text message to confirm enrollment, and receipt of the first text message containing targeted content.

\section{Analysis}

Chi-square tests were used to estimate associations between sociodemographic characteristics and provision of a mobile phone number. A multivariable generalized linear model with a $\log$ link was fit to determine the sociodemographic characteristics associated with enrollment in BBT.

\section{Results}

In 2016, 18,145 unique caregivers received a BB3 toolkit. Of them, 10,843 (60\%) provided a mobile phone number upon receiving the toolkit. Twenty-one percent of caregivers who provided a mobile number completed enrollment in BBT $(\mathrm{N}=2,314)$ (Figure 1). 


\section{Characteristics associated with provision of a mobile number}

Provision of a mobile phone number was significantly associated with language spoken in the home, payer, race/ ethnicity and caregiver marital status. After receiving the BB3 materials, the proportion of caregivers who spoke only English was significantly lower in the group that provided a mobile phone number as compared to the group that did not provide a mobile phone number $(\mathrm{P}<0.0001)$. The proportions of caregivers with private insurance and married caregivers were significantly higher among those who provided a mobile phone number as compared to those who did not provide a mobile phone number $(\mathrm{P}=0.004$ and 0.02 respectively). The proportion of Hispanic caregivers was significantly higher in the group that provided a mobile phone number than in the group that did not provide a mobile phone number $(\mathrm{P}=0.045)$ There were no significant differences in caregiver education and rural vs. urban location between the two groups (Table 1).

\section{Characteristics associated with enrollment in BBT}

In univariate analyses, among caregivers who provided a mobile number, Hispanic and non-White caregivers were less likely to enroll than White non-Hispanic caregivers. Caregivers with public health insurance, or who were uninsured, were less likely to enroll than caregivers private insurance. Caregivers who had not completed high school were less likely to enroll than high school graduates or college graduates. Caregivers who were single were less likely to enroll than married caregivers. Caregivers who spoke only Spanish were less likely to enroll than English speaking caregivers. Caregivers living in rural or frontier areas were less likely to enroll than those living in urban areas (Table 2).

In a multivariable model, among caregivers who provided a mobile number, non-Hispanic White caregivers were more likely to enroll in BBT than Hispanic or nonWhite caregivers (RR 1.15, 95\% CI, 1.04-1.28). Language spoken at home was associated with enrollment and Spanish speaking only caregivers were less likely to enroll in BBT than English speaking only caregivers (RR 0.73, 95\% CI, 0.59-0.90). There was no difference in enrollment between English speaking only caregivers and bilingual caregivers. Caregivers with private insurance were more likely to enroll than caregivers who had public insurance or were uninsured (RR 1.15, 95\% CI, 1.15-1.28). Caregivers who completed college (RR 1.60, 95\% CI, 1.35-1.89) or graduated from high school (RR 1.32, 95\% CI, 1.13-1.54) were more likely to enroll than caregivers who did not graduate from high school. Caregivers living in urban areas were more likely to enroll than caregivers living in rural or frontier areas (RR 1.21, 95\% CI, 1.06-1.37). There was no significant difference in enrollment based on marital status (Table 3).

\section{Discussion}

BB3 successfully reached out to thousands of lower income, minority caregivers in 2016. Many of these caregivers had less than a high school education, were non-English speaking and lived in rural areas. Hundreds of these caregivers enrolled in BBT as a result of these outreach efforts. Despite these successes, these caregivers remained statistically less likely to enroll in BBT than non-minority, English-speaking caregivers with more resources. This is important because traditionally underserved children represented by these populations are at higher risk for poor school readiness and academic achievement, and more likely to live in a home environment that is less supportive of early development $(1,3,6,23)$. Since we excluded caregivers who did not provide a mobile phone number at enrollment from analyses, lack of access to a mobile device does not explain the disparities in enrollment that we found.

Since 2016, BB3 has been working with its community partners to improve outreach efforts to traditionally underserved populations. This is critically important because, despite the rapid growth of mHealth programs targeting the promotion of early child development, there are scant data available about which caregivers are engaging with these programs and which outreach efforts can successfully engage caregivers and children who would most benefit from these interventions. Further research is needed to evaluate whether or not BB3's outreach efforts are successful as well as to identify barriers to enrollment and engagement with BBT and mHealth interventions in general. Possible barriers to engagement may include higher rates of interrupted mobile phone service among caregivers with fewer resources, a higher level of mistrust or stronger desire to preserve privacy among culturally diverse caregivers, or lower technological literacy (24). These findings underscore the need to meaningfully engage and solicit perspectives from lower income and racially diverse groups in the design of similar interventions, and to ensure that strategies for recruitment and enrollment in programs follow best practices for engagement of diverse audiences (25-27). 
Table 1 Description of socio-demographic factors of caregivers who received a BB3 toolkit in $2016(\mathrm{~N}=18,145)$

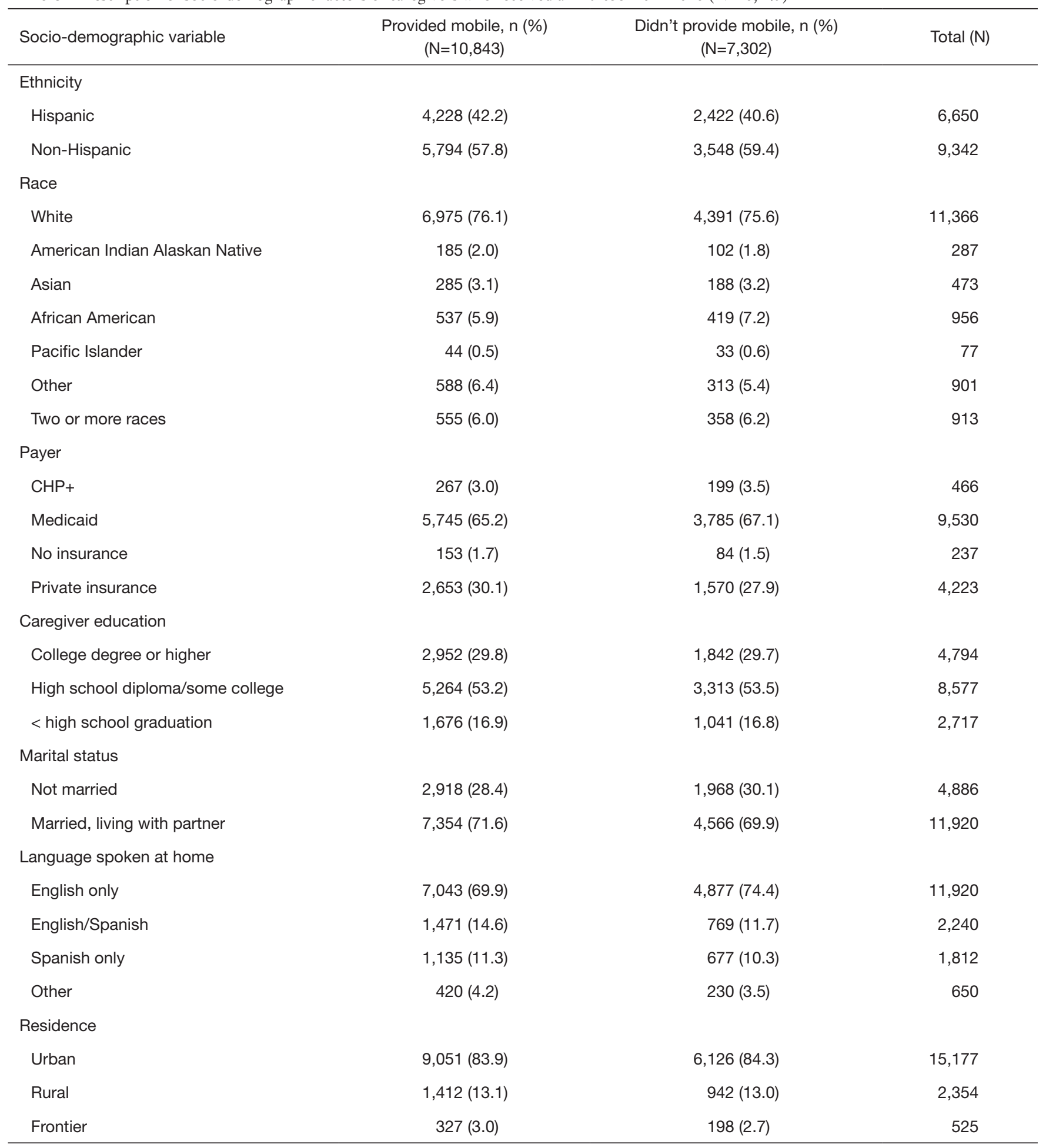

BB3, Bright by Three. 
Table 2 Comparison of socio-demographic factors between caregivers who did and did not enroll in BBT $(\mathrm{N}=10,843)$

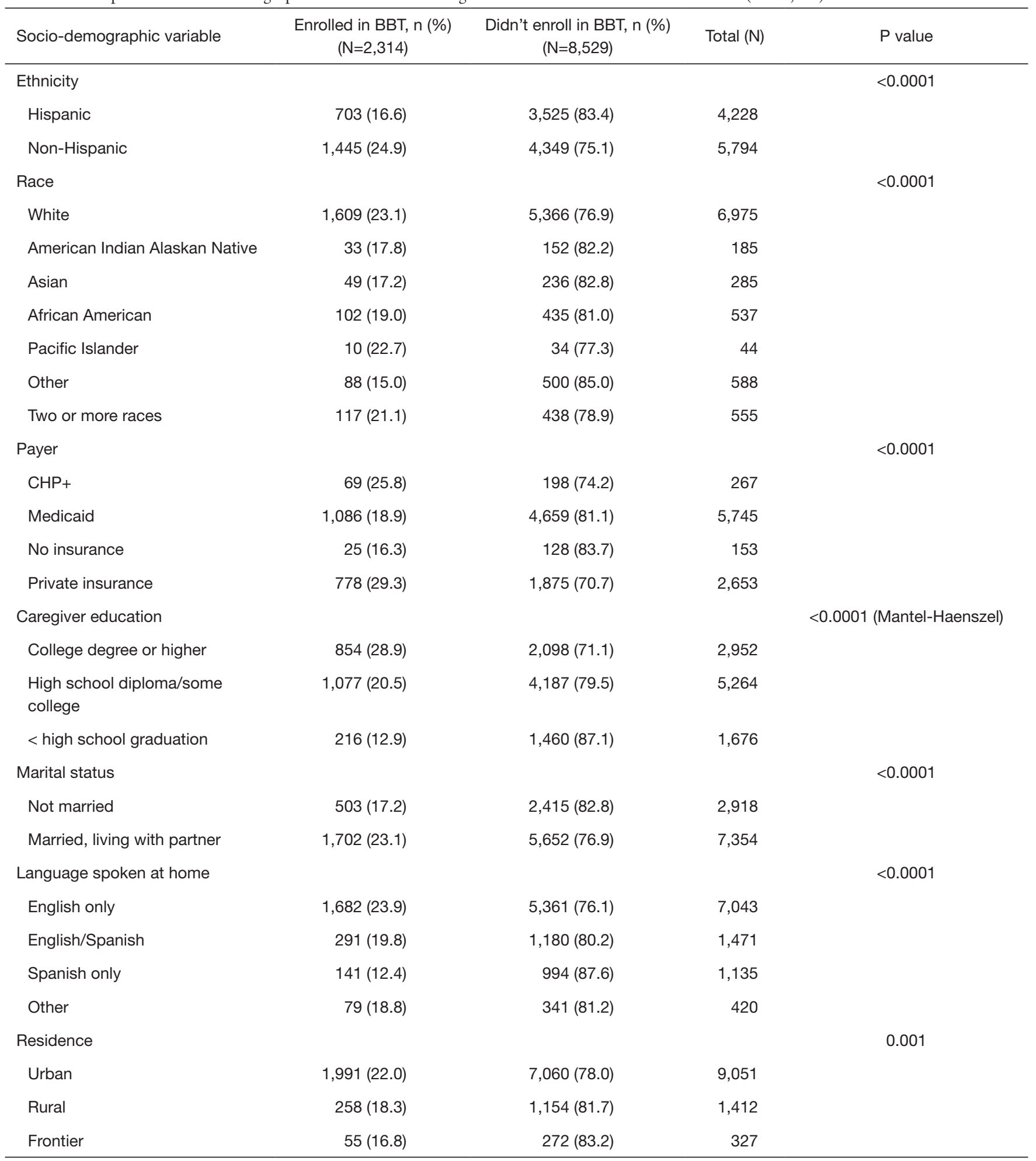

BBT, Bright by Text. 
Table 3 Relative risk of enrollment in BBT based on sociodemographic characteristics

\begin{tabular}{|c|c|c|}
\hline $\begin{array}{l}\text { Total invited to participate in } \\
\text { BBT }(\mathrm{N}=10,843)\end{array}$ & $\begin{array}{l}\text { Relative } \\
\text { risk }\end{array}$ & $\begin{array}{l}95 \% \text { confidence } \\
\text { interval }\end{array}$ \\
\hline \multicolumn{3}{|l|}{ Race/ethnicity } \\
\hline Non-Hispanic White & 1.15 & $1.04-1.28$ \\
\hline Hispanic/non-White & Referent & \\
\hline \multicolumn{3}{|l|}{ Language spoken at home } \\
\hline English only & Referent & \\
\hline Spanish only & 0.73 & $0.59-0.90$ \\
\hline English/Spanish & 1.02 & $0.90-1.17$ \\
\hline English/other & 0.83 & $0.67-1.04$ \\
\hline \multicolumn{3}{|l|}{ Payer } \\
\hline Private & 1.15 & $1.15-1.28$ \\
\hline Public/uninsured & Referent & \\
\hline \multicolumn{3}{|l|}{ Maternal education } \\
\hline College graduation & 1.6 & $1.35-1.89$ \\
\hline $\begin{array}{l}\text { High school graduation/ } \\
\text { some college }\end{array}$ & 1.32 & $1.13-1.54$ \\
\hline $\begin{array}{l}\text { Less than high school } \\
\text { graduation }\end{array}$ & Referent & \\
\hline \multicolumn{3}{|l|}{ Marital status } \\
\hline Married & 1.1 & $0.99-1.23$ \\
\hline Single & Referent & \\
\hline \multicolumn{3}{|l|}{ Residence } \\
\hline Urban & 1.21 & $1.06-1.37$ \\
\hline Rural/frontier & Referent & \\
\hline
\end{tabular}

BBT, Bright by Text.

This is a critical step in improving access to early childhood interventions using mobile solutions and can inform other efforts to use mHealth solutions in health promotion and disease prevention.

This study had some limitations. The BB3 toolkit, and information about BBT, were delivered by community partners in most cases. These partners may have had varied levels of technological literacy themselves or competing program priorities and information to deliver during the visit, thus making fidelity to the delivery of the BB3 materials variable. In 2016, BBT was a new program and unfamiliar to many community partners which likely impacted efforts to promote the program. However, there is expected variability in the delivery of public health interventions and, pragmatically, conformity to a particular script or presentation of materials can never be guaranteed with a high degree of certainty.

\section{Conclusions}

mHealth interventions promoting early childhood development have the potential to reach caregivers across a broad spectrum of the population. However, it cannot be assumed that because of increased access to mobile technology, underserved populations will readily adopt and engage with these interventions. Barriers to adoption and engagement exist among caregivers with lower education and income, minorities, non-English speakers and those living in rural areas. These barriers must be elucidated and addressed before well-meaning interventions can reach their intended audience and effect meaningful public health outcomes. Future research should focus on the identification of barriers to adoption of BBT among target populations, evaluation of efforts to improve outreach and enrollment among traditionally underserved populations, analysis of the socio-demographic characteristics of caregivers enrolling in BBT after exposure to mass advertising efforts, and parental behavior change and developmental outcomes of children after engagement with BBT.

\section{Acknowledgements}

The authors would like to recognize the contributions of Erin McBurney MPH, Carol Gorman BA, Claudia LunaAsturias LGSW, Anowara Begum MPH, an Ashleigh Kirk MSW for their technical and administrative support in preparation of data and this manuscript.

This project was part of a larger project funded by the Piton Foundation; Ben and Lucy Ana Walton Fund of the Walton Family Foundation; The Daniels Fund; The Jack Gantz Foundation; and The Frankenburg-Camp Fund.

\section{Footnote}

Conflicts of Interest: The authors have no conflicts of interest to declare.

Ethical Statement: Approval for this secondary analysis 
of $\mathrm{BB} 3$ program data was obtained from the Colorado Multiple Institution Review Board (COMIRB).

\section{References}

1. Hart B, Risley T. Meaningful Differences in the Everyday Experience of Young American Children. Baltimore: Paul H. Brookes, 1995.

2. Isaacs JB. Starting School at a Disadvantage: The School Readiness of Poor Children. Washington, DC: Brookings Institution Center on Children and Families, 2012.

3. Pati S, Hashim K, Brown B, et al. Early identification of young children at risk for poor academic achievement: preliminary development of a parent-report prediction tool. BMC Health Serv Res 2011;11:197.

4. Felitti VJ, Anda RF, Nordenberg D, et al. Relationship of childhood abuse and household dysfunction to many of the leading causes of death in adults. The Adverse Childhood Experiences (ACE) Study. Am J Prev Med 1998;14:245-58.

5. Garner AS, Shonkoff JP. Early childhood adversity, toxic stress, and the role of the pediatrician: translating developmental science into lifelong health. Pediatrics 2012;129:e224-31.

6. Hair NL, Hanson JL, Wolfe BL, et al. Association of Child Poverty, Brain Development, and Academic Achievement. JAMA Pediatr 2015;169:822-9.

7. Luby J, Belden A, Botteron K, et al. The effects of poverty on childhood brain development: the mediating effect of caregiving and stressful life events. JAMA Pediatr 2013;167:1135-42.

8. Luby JL, Barch DM, Belden A, et al. Maternal support in early childhood predicts larger hippocampal volumes at school age. Proc Natl Acad Sci U S A 2012;109:2854-9.

9. National Scientific Council on the Developing Child. The Science of Early Childhood Development. Cambridge, MA: Harvard University Center on the Developing Child, 2007.

10. University CotDCH. Key Concepts: Toxic Stress. Harvard University. 2018. Available online: https://developingchild. harvard.edu/science/key-concepts/toxic-stress/

11. Campbell FA, Pungello EP, Miller-Johnson S, et al. The development of cognitive and academic abilities: growth curves from an early childhood educational experiment. Dev Psychol 2001;37:231-42.

12. Campbell FA, Ramey FT. Effects of early intervention on intellectual and academic achievement: a follow-up study of children from low-income families. Child Dev 1994;65:684-98.

13. Mendelsohn AL, Mogilner LN, Dreyer BP, et al. The impact of a clinic-based literacy intervention on language development in inner-city preschool children. Pediatrics 2001;107:130-4.

14. Muennig P, Robertson D, Johnson G, et al. The Effect of an Early Education Program on Adult Health: The Carolina Abecedarian Project Randomized Controlled Trial. Am J Public Health 2011;101:512-6.

15. Weitzman CC, Roy L, Walls T, et al. More evidence for reach out and read: a home-based study. Pediatrics 2004;113:1248-53.

16. Center PR. Mobile Fact Sheet. Washington, DC. 2018. Available online: http://www.pewinternet.org/fact-sheet/ mobile/. Accessed 4/25/2018.

17. Bull S. Beyond acceptability and feasibility: moving mHealth into impact. Mhealth 2016;2:45.

18. Olson KB, Wilkinson CL, Wilkinson MJ, et al. Texts for Talking: Evaluation of a Mobile Health Program Addressing Speech and Language Delay. Clin Pediatr (Phila) 2016;55:1044-9.

19. York BN, Loeb S. One Step at a Time: The Effects of an Early Literacy Text Messaging Program for Parents of Preschoolers. In: Research NBoE. editor. Cambridge, MA, 2014.

20. Camp B, Frederick B. My First Picture Book. Baltimore, MD: Brookes Publishing Co., 1999.

21. Camp BW. Language Power. Baltimore, MD: Brookes Publishing Co., 1999.

22. Sparling J, Lewis I. Learning Games. Washington, DC: Teaching Strategies, Inc., 2008.

23. Hillemeier MM, Morgan PL, Farkas G, et al. Perinatal and socioeconomic risk factors for variable and persistent cognitive delay at 24 and 48 months of age in a national sample. Matern Child Health J 2011;15:1001-10.

24. Newman L, Biedrzycki K, Baum F. Digital technology use among disadvantaged Australians: implications for equitable consumer participation in digitally-mediated communication and information exchange with health services. Aust Health Rev 2012;36:125-9.

25. Buis LR, Hirzel L, Turske SA, et al. Use of a text message program to raise type 2 diabetes risk awareness and promote health behavior change (part I): assessment of participant reach and adoption. J Med Internet Res 2013;15:e281. 
26. Christopoulos KA, Cunningham WE, Beckwith CG, et al. Lessons Learned From the Implementation of Seek, Test, Treat, Retain Interventions Using Mobile Phones and Text Messaging to Improve Engagement in HIV Care for Vulnerable Populations in the United States. AIDS Behav

doi: $10.21037 /$ mhealth.2018.11.03

Cite this article as: Cunningham $M$, Bull S, McNulty MC, Colborn K, Chavez C, Berman S, McSpadden J, Wigdor $\mathrm{J}$, Allison MA. Does a text-messaging program to promote early childhood development reach the highest risk families? mHealth 2018;4:55.
2017;21:3182-93.

27. Dobson R, Whittaker R, Bartley H, et al. Development of a Culturally Tailored Text Message Maternal Health Program: TextMATCH. JMIR Mhealth Uhealth 2017;5:e49. 